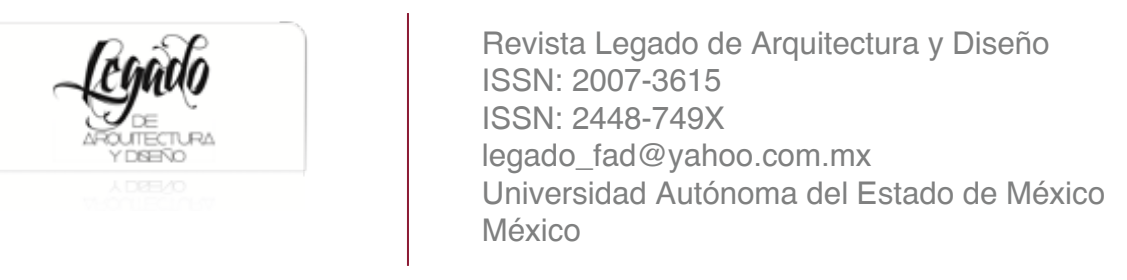

\title{
VIVIENDA TRADICIONAL DE TIERRA CALIENTE: UN ESTUDIO DE CASO REGIONAL MICHOACANO
}

Ayala-Ortega, Luis Alfredo; Azevedo-Salomao, Eugenia María

VIVIENDA TRADICIONAL DE TIERRA CALIENTE: UN ESTUDIO DE CASO REGIONAL MICHOACANO

Revista Legado de Arquitectura y Diseño, vol. 2019, núm. 26, 2019

Universidad Autónoma del Estado de México, México

Disponible en: http://www.redalyc.org/articulo.oa?id=477961406008

Esta obra está bajo una Licencia Creative Commons Atribución-NoComercial-SinDerivar 4.0 Internacional. 


\title{
VIVIENDA TRADICIONAL DE TIERRA CALIENTE: UN ESTUDIO DE CASO REGIONAL MICHOACANO
}

\author{
TRADITIONAL HOUSING OF TIERRA CALIENTE: A \\ MICHOACAN REGIONAL CASE STUDY
}

\author{
Luis Alfredo Ayala-Ortega tecnologik@hotmail.com \\ Universidad Michoacana de San Nicolás de Hidalgo, México \\ Eugenia María Azevedo-Salomao \\ eazevedosa@yahoo.com.mx \\ Universidad Michoacana de San Nicolás de Hidalgo, México
}

Revista Legado de Arquitectura y Diseño,

Universidad Autónoma del Estado de México, México

Recepción: 28 Febrero 2019 Aprobación: 03 Junio 2019

Redalyc: http://www.redalyc.org/ articulo.oa?id $=477961406008$
Resumen: En el territorio de Michoacán, la diversidad de climas ha permitido, desde una larga temporalidad, adaptaciones de la vivienda congruentes a esas condiciones y necesidades climáticas. Los recursos materiales como la piedra, tierra, madera y otros complementos vegetales, se han utilizado para resolver la habitabilidad en función del medio físico geográfico y modos de vida.

El interés de esta comunicación es abordar el estudio de la tradición constructiva de la vivienda en la región de la Depresión Balsas-Tepalcatepec en el sureste del estado de Michoacán, conocida como "Tierra Caliente". El estudio se centra en el poblado de Carácuaro de Morelos, localidad que en su fisonomía arquitectónica presenta los elementos constructivos característicos de esta región.

Para explicar las características constructivas propias del lugar seleccionado, el trabajo se apoya en fuentes documentales, así como del contacto directo con la arquitectura del lugar. Para ejemplificar la tradición constructiva del sitio elegido, se seleccionó una vivienda del primer cuadro del asentamiento. Además de la revisión de la materialidad de la vivienda -caso de estudio- el acercamiento con los habitantes permitió la consolidación del conocimiento histórico constructivo de esta vivienda.

Los resultados de esta investigación se materializan en una serie de levantamientos de los materiales, sistemas constructivos y de las respuestas estructurales, que ayudan a dilucidar sobre el proceso de adaptación de la vivienda en una localidad con clima caluroso. Se concluye reflexionando sobre las condiciones de sostenibilidad actuales de la vivienda tradicional, que permiten su permanencia o cambio.

Palabras clave: adaptación, clima cálido, tradición, vivienda.

Abstract: In the territory of Michoacan, the diversity of climates has allowed, from a long period of time, adaptations of housing congruent to those conditions and climatic needs. The material resources such as stone, earth, wood and other vegetal complements, have been used to solve the habitability in function of the geographic physical means and ways of life.

The interest of this communication is to address the study of the constructive tradition of housing in the region of the Depression Balsas-Tepalcatepec in the southeast of the State of Michoacan, known as "Tierra Caliente". The study focuses on the town of Carácuaro de Morelos, a town that in its architectural appearance presents the constructive elements characteristic of this region.

To explain the constructive characteristics of the selected site, the work is supported by documentary sources, as well as direct contact with the architecture of the place. To illustrate the constructive tradition of the chosen site, a dwelling was selected from the first square of the settlement. In addition to the review of the materiality of the house - study case - the approach with the inhabitants allowed the consolidation of the constructive historical knowledge of this house. 
The results of this research are materialized in a series of surveys of materials, construction systems and structural responses, which help to elucidate about the adaptation process of housing in a hot climate location. It concludes by reflecting on the current sustainability conditions of traditional housing, which allow its permanence or change.

Keywords: adaptation, tradition, dwelling, hot climate.

\section{INTRODUCCIÓN}

En el estado de Michoacán, la arquitectura de tierra representa la mayoría de las expresiones tradicionales de construcción, lo que subraya la importancia de indagar en el conocimiento histórico-constructivo de esta tipología material.

Las reflexiones expuestas retoman el tema del patrimonio construido de tierra en Michoacán, enfocándose en aspectos de la materialidad, así como los hábitos sociales, usos y adecuación al medio ambiente, en un caso de estudio representativo de esta arquitectura.

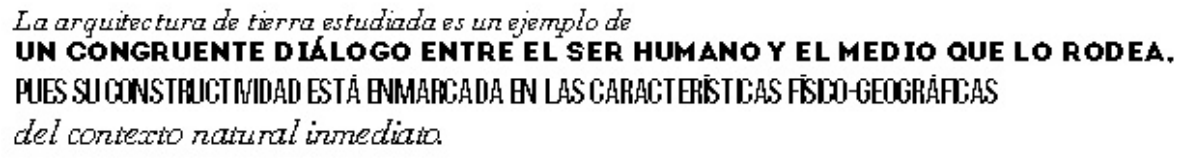

La arquitectura de tierra estudiada es un ejemplo de un congruente diálogo entre el ser humano y el medio que lo rodea, pues su constructividad está enmarcada en las características físico-geográficas del contexto natural inmediato.

El territorio michoacano cuenta con una gran diversidad de ejemplos de esta tradición constructiva, que de acuerdo con la región en que se ubican expresan diferentes soluciones. En el estado de Michoacán se observan cuatro regiones geográfica-culturales: Eje Volcánico Transversal, Bajíos y Serranías del norte, Depresión BalsasTepalcatepec y la Sierra Madre del sur y Costa (Guzmán, 2007: 6-7).

Si bien, la tierra está presente en muchas expresiones arquitectónicas, para esta comunicación interesa la concreción en el género habitacional, en una zona poco abordada a pesar de ser rica en manifestaciones culturales únicas en el estado de Michoacán : la región conocida como “Tierra Caliente”. Dicha región se ha caracterizado por contar con un ambiente y clima agreste, que ha moldeado la vida en función de sus actividades económicas - agrónomas - y que su aislamiento de grandes núcleos urbanos ha permitido la conformación de una imagen y arquitectura particular, acorde a sus valores culturales, necesidades y formas de vida.

En particular, se analiza la localidad de Carácuaro de Morelos, cabecera del municipio homónimo, a través del estudio de una vivienda dentro del primer cuadro del asentamiento, como un ejemplo representativo de esta arquitectura regional tierracalentana. 


\section{ANTECEDENTES: EL MEDIO FÍSICO GEOGRÁFICO CULTURAL DE TIERRA CALIENTE}

La zona de las Depresiones Balsas y Tepalcatepec es una amplia región de tierras bajas situadas entre el Eje Neovolcánico y la Sierra Madre del Sur. Dicha depresión ocupa porciones de los estados de Michoacán, Guerrero, Morelos y Puebla. Para el presente trabajo se estudia la región de la Depresión Balsas-Tepalcatepec, en la porción comprendida en el estado de Michoacán, y conocida como Tierra Caliente. Esta región de Tierra Caliente abarca dos zonas geográficas claramente distinguibles: el Valle de Apatzingán y el Sureste del Estado.

Este trabajo aborda el estudio de las manifestaciones arquitectónicas en la región del sureste, en la Depresión del Balsas, territorio que comprende los municipios de Carácuaro, Churumuco, Huetamo de Núñez, Nocupétaro, San Lucas, Tafetán, Tiquicheo de Nicolás Romero, Turicato y Tuzantla.

La Depresión del Balsas es una región agreste, árida, con un clima extremo, llegando a superar los $50^{\circ} \mathrm{C}$ en verano y disminuyendo hasta los $10{ }^{\circ} \mathrm{C}$ en invierno, con marcados periodos de lluvia, que indican la pauta del cultivo y cosecha del campo; la ubicación geográfica de algunos de los municipios de esta zona, propició que su desarrollo se vea limitado ; por otra parte , el paisaje físico es dominado por pastizales secos, huizaches , cactáceas y maderas duras autóctonas de la región como son parotas, tepehuajes y cueramos.

En este contexto físico geográfico, la vivienda tradicional se adaptó al clima cálido. Con materiales locales se construyeron viviendas de adobe con cubiertas inclinadas, pero ligeras, hechas de madera rolliza delgada y de carrizos para permitir la penetración de ventilación natural. Además de la tipología constructiva de vivienda de adobe también se encuentra en la región el sistema constructivo de bajareque : estructuras realizadas con horcones de madera para formar apoyos verticales, entre cada horcón se encuentran rollizos de madera con varas entretejidas de forma horizontal ; los muros aplanados con lodo combinado con paja y la cubierta de dos vertientes recubierta de paja, palma o teja (Torres, 2008: 104, citado en Azevedo, 2008a).

\section{EL ASENTAMIENTO CARÁCUARO DE MORELOS}

SELECCIONADO:

El origen y posterior consolidación de este poblado se remontan a la época prehispánica, atravesando etapas históricas que lo han amalgamado, desde la llegada de la orden religiosa de los agustinos a la región de Tierra Caliente en 1537.

Esta población también está vinculada al caudillo independentista José María Morelos y Pavón, quien en 1799 cubrió un interinato como cura de la Parroquia de San Agustín y vivió en el asentamiento antes de unirse a los movimientos independentistas gestados por Miguel Hidalgo y Costilla. A 
la llegada de Morelos, éste hace una descripción de la situación con la que se encontró al llegar al Curato, según lo relata Herrera Peña:

Al llegar a la cabecera de su curato, el hombre hace el necesario reconocimiento del terreno. Hay tres pueblos en su jurisdicción: Carácuaro, Nocupétaro y Acuyo. Los dos primeros están situados “a una legua de distancia”, uno frente al otro, río de por medio. Su apariencia no difiere mucho de los otros de la Tierra Caliente: casas en desorden con techos de paja, "interpoladas" con árboles secos, sin sombra, de distintas variedades, y sin calles. Es un pueblo como muchos que todavía pueden verse en esa región (1995: 277).

Con relación a las características físicas; está moldeado por su relieve, conjuntándose elementos naturales como la topografía y el cauce del río que lo atraviesa de noreste a suroeste, configurando así su asentamiento; al respecto Juan Francisco Meza Aguilar dice sobre el acceso a éste: "dicho trayecto conduce al núcleo urbano histórico que se localiza hacia un meandro del mencionado río, situación que le otorga una singular imagen contextual" (Meza, 449, citado en Guzmán 2007) (figura 1).

Esta configuración urbana-paisajística ha sido producto de la manipulación del humano sobre el territorio, resultando en un paisaje antropizado diseñado, donde se observa en mayor o menor medida un sentido de ordenamiento y planeación del asentamiento, adecuándose a las características geomorfológicas del mismo (Gracia, 2009).

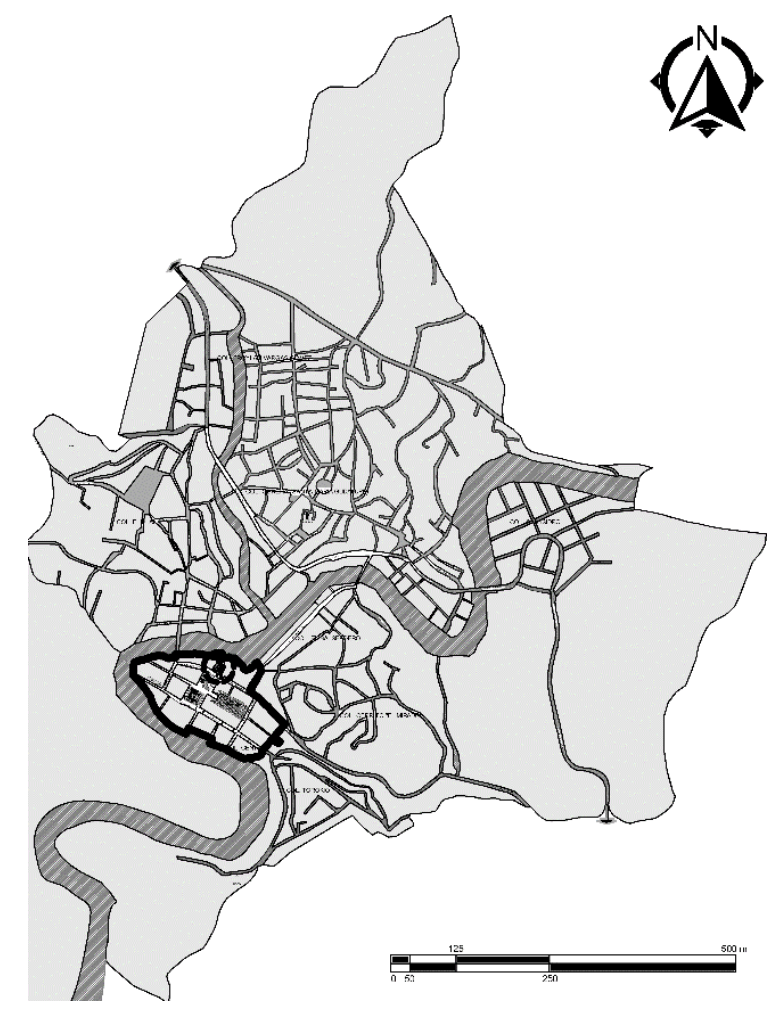

Figura 1. Localización del centro de población respecto al territorio de la localidad de Carácuaro de Morelos, con relación a su contexto natural.

Fuente: Elaboración propia.

Esta relación, topografía y río, se puede inferir, ha influido significativamente en la configuración del partido arquitectónico de las viviendas, en específico, en la disposición de vanos, y ubicación de los 
espacios habitables, que a la postre generan condiciones higrotérmicas muy particulares dentro de la vivienda.

\section{Este condicionamiento del río, \\ PROVOCA QUE LA ORTOGONALIDAD DE LA TRAZA}

sey aya perdiendo enie más se aleja del primer cuadro,

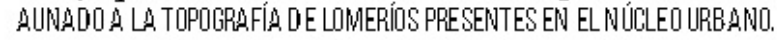

Sobre la traza del núcleo fundacional de Carácuaro de Morelos, ésta es regular , cuasi reticular, la cual está circundada por el cauce del río homónimo, lo que se ve manifestado en las vialidades que corren transversales al templo parroquial, y que están orientadas de norte a sur y son de longitud más cortas, donde algunas de ellas no cuentan con nomenclatura. Este condicionamio del río, provoca que la ortogonalidad de la traza se vaya perdiendo entre más se aleja del primer cuadro , aunado a la topografía de lomeríos presentes en el núcleo urbano.

Sobre la posible detección de una lógica en la distribución de los inmuebles , así como de sus fachadas sobre los solares debido a la trayectoria solar (figura 2), no se han encontrado evidencias que apunten a esto, más bien, se in fiere, que la distribución del asentamiento obedece a una disposición con relación a la Parroquia, hito de mayor referencia urbana en la localidad.

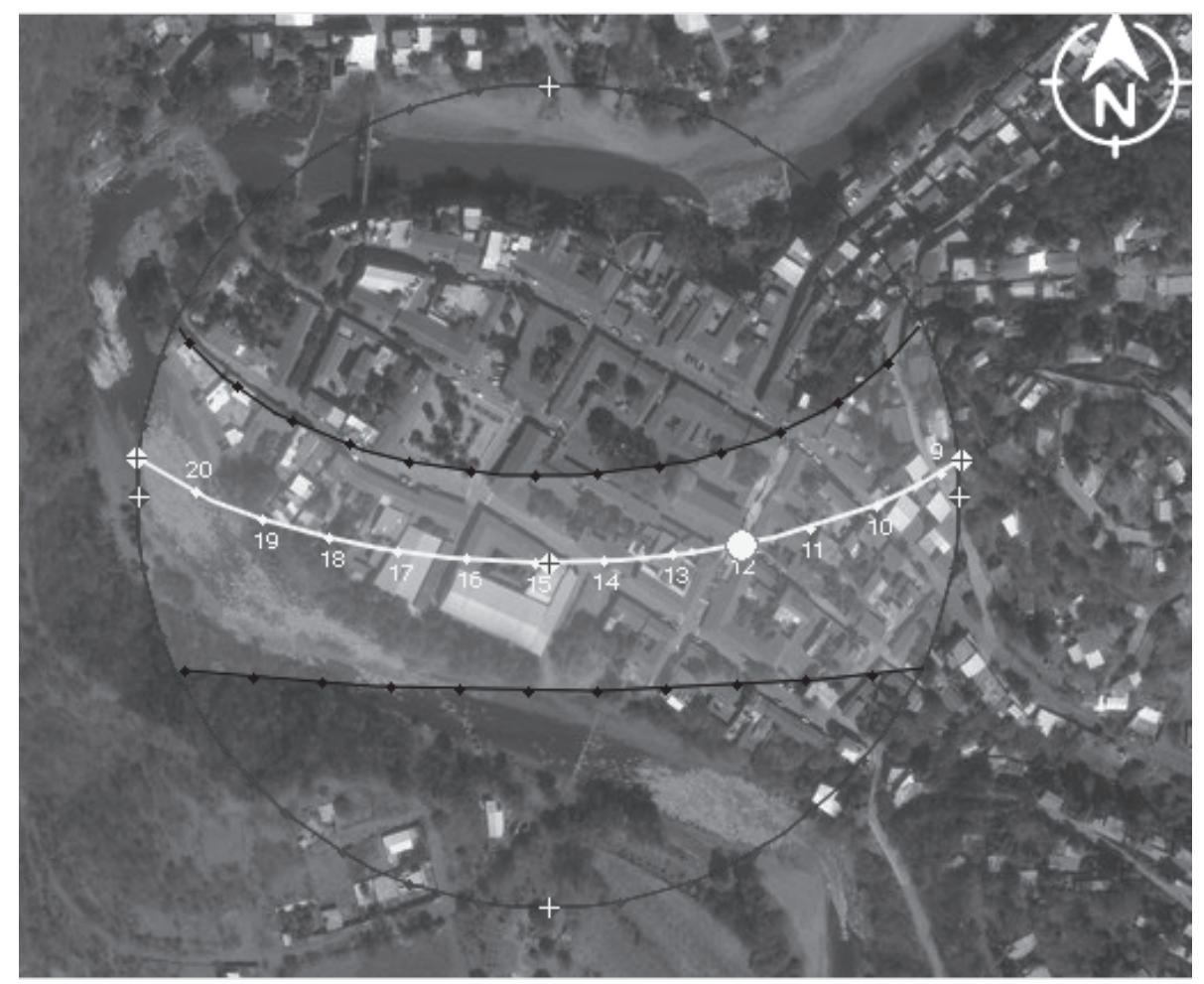

Figura 2. Posición y trayectoria del recorrido solar sobre el asentamiento de Carácuaro de Morelos. Fuente: Elaboración propia con base en SunEarthTools (2019). 


\section{CARACTERIZACIÓN CLIMÁTICA DEL SITIO}

Todo clima, a saber, hallase en relación directa al régimen térmico, la presión barométrica que influye en definitiva sobre los vientos dominantes y la precipitación pluvial concomitante.

En la tabla 1 se presentan las características y condiciones climáticas del territorio del municipio de Carácuaro.

Tabla 1. Condiciones climáticas del municipio de Carácuaro.

\begin{tabular}{|l|l|l|l|l|l|l|l|l|l|}
\hline Localiaad & $\begin{array}{l}\text { Altitud } \\
(m s n m)\end{array}$ & $\begin{array}{l}R H \\
(\%)\end{array}$ & $\begin{array}{l}R R \\
(\mathrm{~mm})\end{array}$ & $\begin{array}{l}\text { Rango de } \\
\text { Precipitación } \\
(\mathrm{mm})\end{array}$ & $\begin{array}{l}\text { RD } \\
\text { anual } \\
(\text { dáias })\end{array}$ & $\begin{array}{l}\text { Temperatura } \\
\text { promedio } \\
\text { anual } \\
\left({ }^{\circ} \mathrm{C}\right)\end{array}$ & $\begin{array}{l}\text { Rango } \\
\text { temperatura } \\
\left({ }^{\circ} \mathrm{C}\right)\end{array}$ & Mes más frio & $\begin{array}{l}\text { Mes más } \\
\text { cálido }\end{array}$ \\
\hline $\begin{array}{l}\text { Carácuaro } \\
\text { de } \\
\text { Morelos }\end{array}$ & 534 & 62 & 902 & $700-1000$ & 55.9 & 25.9 & $22-28^{\circ}$ & $\begin{array}{l}\text { Febrero-Marzo } \\
11.5^{\circ}\end{array}$ & $\begin{array}{l}\text { Mayo } \\
36.8^{\circ}\end{array}$ \\
\hline
\end{tabular}

Fuente: Elaboración propia con base en INEGI (2009) y datos arrojados por software Meteonorm.

Además de estas variables, los vientos dominantes y la radiación solar parten como factores que inciden en el desempeño de la vivienda, como dispositivo de adaptación al medio. Sobre los vientos dominantes, para el caso del asentamiento estudiado, la tendencia de los vientos dominantes son los que provienen del suroeste, con velocidades de $40 \mathrm{~km} / \mathrm{h}$, con una presencia durante un intervalo de menos de 55 horas al año, es decir, en esta orientación se presentan los vientos más fuertes, sin embargo, su permanencia durante el año es la más corta. Por otro lado, al norte, en el rango de los $10-15 \mathrm{~km} / \mathrm{h}$ está presente la periodicidad más larga de los vientos al año, con 553 o más horas al año (figura 3).
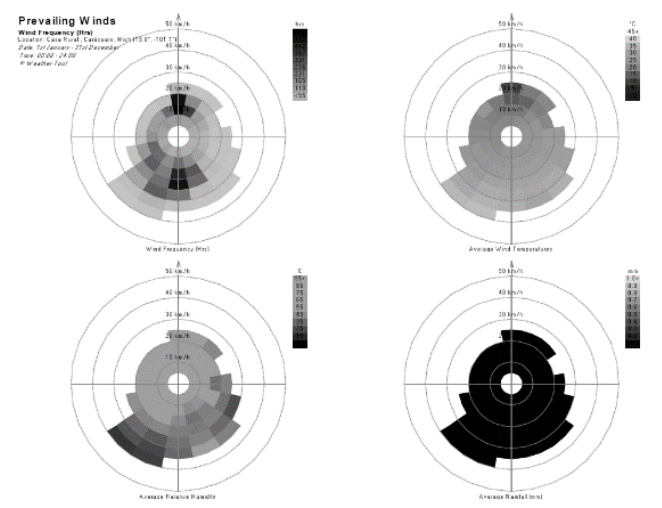

Figura 3. Gráficas de dirección, velocidad, horas y temperatura de vientos dominantes en el municipio de Carácuaro, Michoacán.

Fuente: Elaboración propia con base en software Autodesk Ecotect.

Aunque el suroeste ofrece los vientos con mayor velocidad, no así su periodicidad durante el año. Es en esta orientación, que estos son justamente los más cálidos, presentado unas temperaturas promedio de entre 35 y $40{ }^{\circ} \mathrm{C}$, mientras que, al norte, se presentan los más fríos con temperaturas de $10^{\circ} \mathrm{C}$ las más bajas.

Lo anterior se traduce en que, para el caso de los inmuebles ubicados dentro del primer cuadro, éstos se ven afectados por los vientos 
dominantes provenientes del suroeste (continua), norte (punteada) y noroeste (segmentada), siendo los primeros los de mayor intensidad, mientras que los provenientes del norte son los más fríos, aunque a una velocidad promedio de $20 \mathrm{~km} / \mathrm{h}$, y los últimos los que poseen mayor concentración de humedad , 75\% - 85\%, pero que mantienen una velocidad relativamente baja $-20 \mathrm{~km} / \mathrm{h}$. En cuanto al asolamiento, como agente de influencia en la orientación de las viviendas, la presencia de portales y la distribución de espacios interiores, amortiguan la incidencia solar; más adelante se estudia el caso de la vivienda seleccionada en lo referente a la adecuación al medio ambiente.

\section{CARACTERÍSTICAS ARQUITECTÓNICAS DE LA VIVIENDA}

A decir de Ambrosio Ochoa Cabrera, la localidad de Carácuaro junto con los municipios de Nocupétaro, Huetamo, Purechucho y San Lucas forman parte de "el corredor principal de Tierra Caliente", el cual históricamente permitió la comunicación entre esta región y la capital, camino que actualmente se mantiene casi inalterable y que además atraviesa la franja compuesta por los territorios de tierra fría -Tiripetío, Acuitzio del Canje y Villa Madero - con los de Tierra Caliente (Ochoa, 2003: 205).

Para entender la forma de generación de la arquitectura doméstica tradicional regional, hay que entender cómo ha sido su proceso histórico - cultural, pues como dice Eugenia Azevedo "no hay que olvidar que cada edificio nuevo construido surge de otros ya existentes, de ahí no se pueden olvidar el rol de la tradición en el acto de configuración arquitectural" (Azevedo, 2008b: 20).

Debido a su aislamiento - a causa de falta de infraestructura carretera y difícil topografía- la vida económica de esta región se ha visto reducida a la explotación del campo -agricultura y ganadería- a pesar de que la productividad se vea condicionado por los marcados periodos estacionarios de lluvia, escasa disposición de cuerpos de agua y un suelo, muchas veces poco apto para la siembra -en el caso de Nocupétaro, está compuesto en su gran mayoría por estratos de arena, y para la localidad de Carácuaro de Morelos, su difícil orografía compuesta de piedra laja, aunado a otros factores, ocasionan que estas poblaciones presenten los índices de pobreza extrema más altos en el territorio michoacano.

\section{Los poblados, rancherías ycomunidades de \\ LA REGIÓN COMPARTEN UNA IMAGEN CARACTERÍSTICA, \\ debido a las condiciones climátic as imperantes del medio, \\ DANDO LIGAR A UNA ARQUITECTURA DEADOBE, MADERA Y TEEA.}

Los poblados, rancherías y comunidades de la región comparten una imagen característica, debido a las condiciones climáticas imperantes del medio, dando lugar a una arquitectura de adobe, madera y teja. Estas características constructivas señaladas con anterioridad están sujetas a la 
disposición de recursos naturales que el árido medio natural provee, con soluciones específicas acordes al clima cálido.

En estas comunidades, es observable el uso de materiales propios de la región sin un gran proceso de manufactura, esto es, el uso y disposición de estos casi en su forma natural (figura 4).

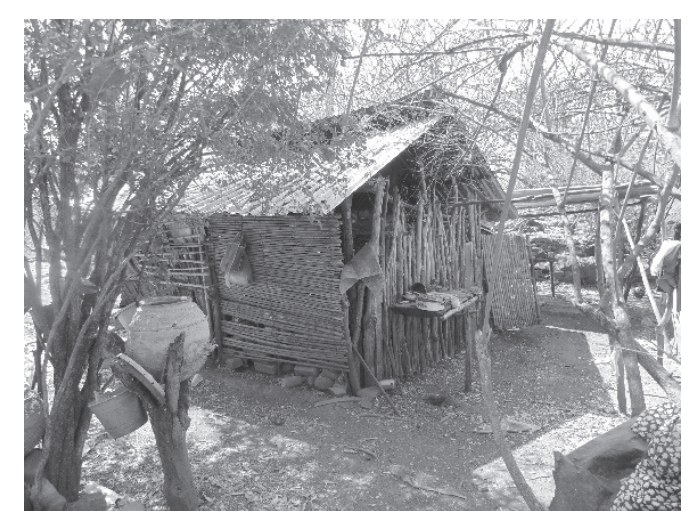

Figura 4. Ejemplo de vivienda de la comunidad de "El Zapote de los Gómez” en la región de Tierra Caliente.

Fuente: Fotografías: archivo personal.

Así mismo, a pesar de que poco a poco la arquitectura, y en particular la vivienda ha ido perdiendo la esencia tan característica del patrimonio vernáculo ante fenómenos contemporáneos, existe una serie de permanencias "en lo concerniente a soluciones constructivas y espaciales; en muchos casos, no obstante, el cambio en los materiales utilizados, las soluciones del espacio se mantuvieron, aspecto atribuible también a la permanencia en la forma de habitar" (Torres, 2008: 27).

\section{LA VIVIENDA CASO DE ESTUDIO}

En orden de ejemplificar la tradición constructiva del sitio se seleccionó una vivienda ubicada dentro del primer cuadro del núcleo urbano histórico de la población de Carácuaro.

Dicho inmueble se trata de una vivienda unifamiliar, de un solo nivel, con portal exterior, ubicada dentro del primer cuadro del asentamiento, de inicios del siglo Xx, que, en su historia, ha sido objeto de múltiples cambios de uso, sin embargo, no han implicado en grandes alteraciones del esquema arquitectónico original; lo que demuestra que ha sido un inmueble en constante uso, lo que quizás explique su excelente estado de conservación, con mínimas evidencias de deterioros, y si, con alteraciones, pero las normales dentro de los cambios cronológicos de las dinámicas de vida (figura 5). 


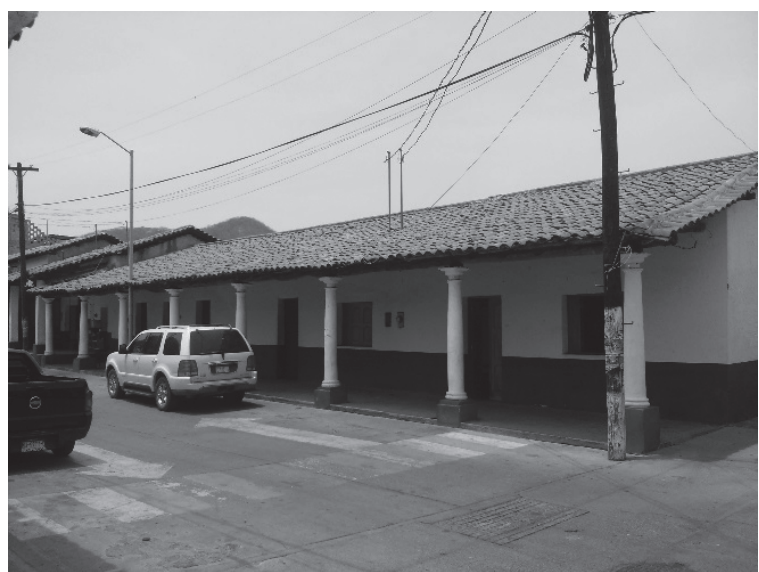

Figura 5. Fachada principal del inmueble seleccionado.

Fuente: Fotografía: archivo personal.

\section{PARTIDO ARQUITECTÓNICO}

La fachada principal está orientada al suroeste compuesta por un portal exterior que funge como recibidor, posterior y en paralelo a éste se encuentra la crujía principal, en la cual en sus accesorias y habitaciones se encuentran ocupando estos espacios un billar, una habitación reconvertida y el zaguán (figura 6). 


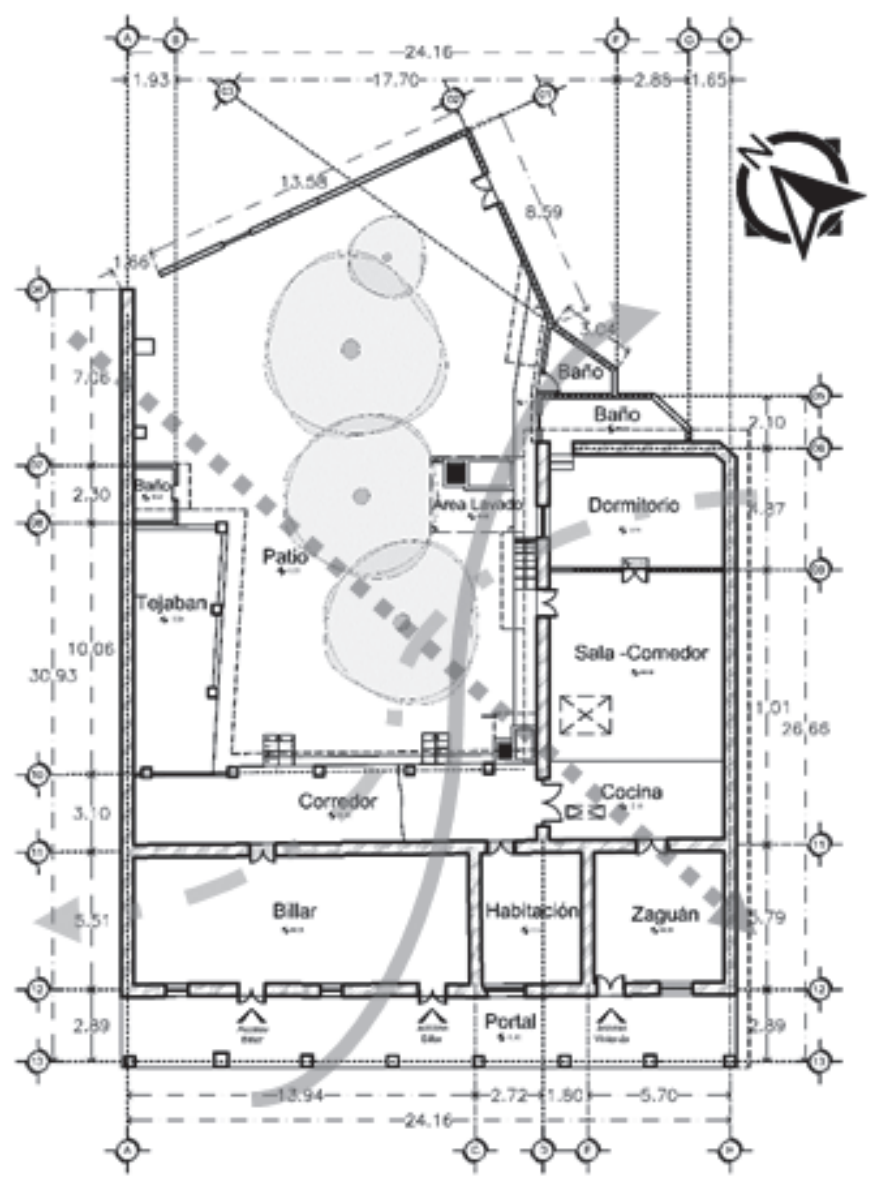

Figura 6. Planta Arquitectónica de la vivienda estudiada, con las incidencias y direcciones de los vientos dominantes. NOTA: FLECHA SÓLIDA (VIENTOS MÁS CÁLIDOS CON TEMPERATURAS ENTRE 30 Y $35^{\circ} \mathrm{C}$ Y CON VELOCIDADES DE $35 \mathrm{KM} / \mathrm{H}$ ), FLECHA PUNTEADA (VIENTOS MÁS FRÍOS CON TEMPERATURAS ENTRE $10 \mathrm{Y} 15^{\circ} \mathrm{C}$ Y CON UNA VELOCIDAD DE $25 \mathrm{KM} / \mathrm{H}$ ), FLECHA SEGMENTADA (VIENTOS DOMINANTES CON MAYOR PORCENTAJE DE HUMEDAD, ENTRE 75 Y 85\% Y CON UNA FUERZA DE 20 KM/H).

Fuente: Elaboración propia.

El zaguán, ubicado en el extremo este de la fachada, conduce a la segunda crujía, la cual está distribuida perpendicularmente al cuerpo de la crujía principal, y que alberga los espacios de la cocina, sala-comedor y dormitorio principal.

$\mathrm{Al}$ noroeste de este recinto, se encuentra el corredor interno que rodea y conduce a su vez al patio interior y a un tejaban, donde se almacenan todos los enseres del campo de los propietarios.

Al centro del solar, se ubica el patio de forma irregular, el cual está $f$ anqueado por una serie de servicios complementarios a la vivienda - múltiples baños y área de lavado.

\section{ESPACIALIDAD}

Dentro de la diversidad de ejemplos de viviendas, que por sus particularidades pueden caracterizarse como tradicionales por su resistencia cultural ante las influencias de la modernidad, en este contexto 
se pueden identificar dos patrones de configuración de la vivienda que se puede observar, no únicamente en los núcleos urbanos, sino en la arquitectura rural de comunidades y rancherías.

El primero de estos corresponde, en su gran mayoría a los que se encuentran en el primer cuadro de la localidad de Carácuaro de Morelos -como el caso de estudio, que son viviendas de mayor dimensión y que en su fachada presentan portales que dan hacia el exterior.

La segunda tipología, son viviendas, que no poseen portal exterior, sino que únicamente en su fachada es observable el paramento del muro, con sus vanos claramente identificables y su alero prolongado; en estos ejemplos, se accede por un zaguán, con habitaciones a los costados, y con una distribución de portales en torno a un patio central (figura 7).

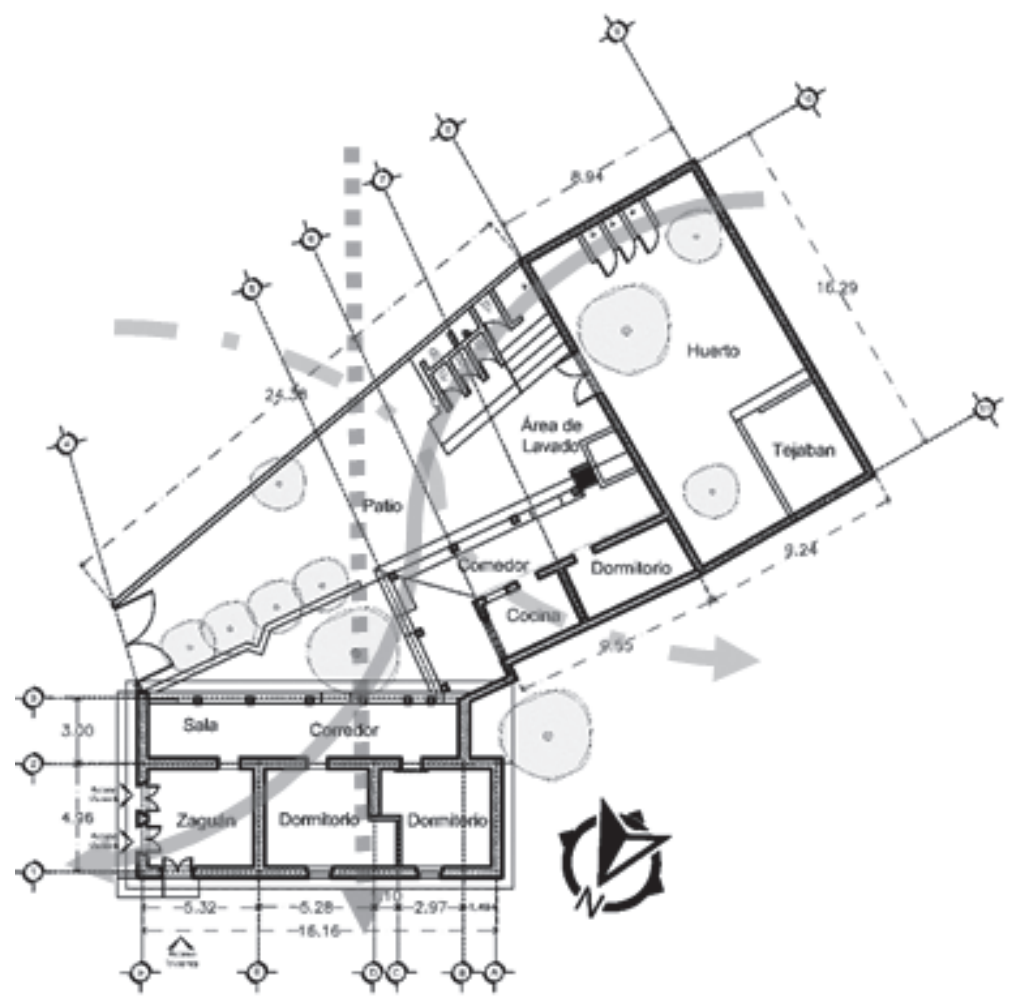

Figura 7. Planta Arquitectónica de vivienda con segunda tipología espacial, con la incidencia y dirección de los vientos dominantes.

NOTA: FLECHA SÓLIDA (VIENTOS MÁS CÁLIDOS CON TEMPERATURAS ENTRE 30 Y $35^{\circ} \mathrm{C}$ Y CON VELOCIDADES DE $35 \mathrm{KM} / \mathrm{H}$ ), FLECHA PUNTEADA (VIENTOS MÁS FRÍOS CON TEMPERATURAS ENTRE $10^{\circ} \mathrm{C} Y 15^{\circ} \mathrm{C}$ Y CON UNA VELOCIDAD DE $25 \mathrm{KM} / \mathrm{H}$ ), FLECHA SEGMENTADA (VIENTOS DOMINANTES CON MAYOR PORCENTAJE DE HUMEDAD, ENTRE 75 Y 85\% Y CON UNA FUERZA DE 20 KM/H).

Fuente: Elaboración propia.

La presencia de portales, al frente presentes en la tipología I, no solo es un elemento jerarquizador dentro del asentamiento urbano, sino un espacio de encuentro social y un dispositivo de control ambiental, de ahí que permita limitar el contacto directo entre la radiación solar y los paramentos de los espacios privados internos, proyectando sombra sobre esto; además, la inclinación de la cubierta que cubre los portales permite aminorar la fuerza de los vientos sin bloquear el paso de corrientes de aire, por lo que la intensidad de los vientos al interior de los espacios abiertos 
- patios- será mucho menor. No así, en el caso de la tipología II, donde el paramento queda expuesto directamente a las variables climáticas.

Para el caso de la vivienda analizada, presenta una orientación de su fachada principal hacia el suroeste, que de acuerdo con la gráfica estereográfica (figura 8), y con datos del $1^{\circ}$ de abril de 2019, a las 12:00 pm., el sol se encuentra en este periodo a una altitud de $71.0^{\circ}$ con un azimut de $140.2^{\circ}$, por lo que en este punto, todavía el sol no incide directamente perpendicular al plano terrestre, cuya incidencia en la vivienda se da en su fachada lateral, la cual se ubica al sureste.

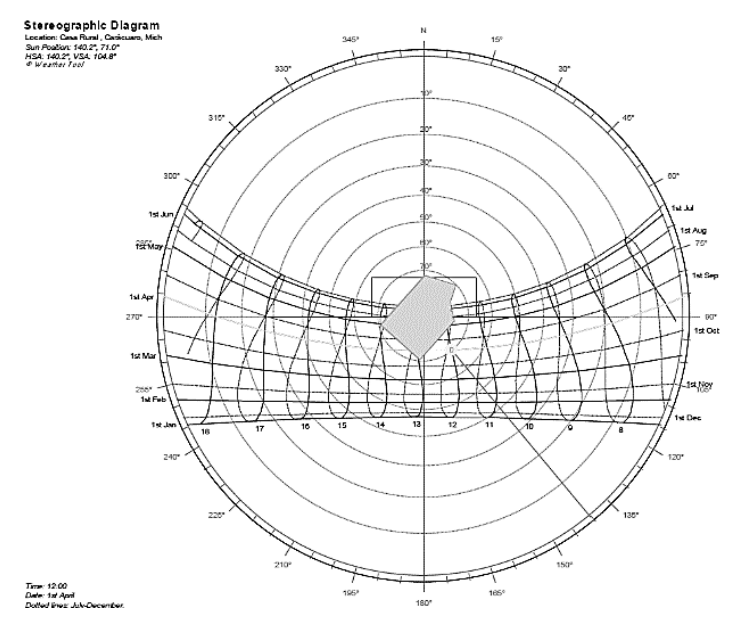

Figura 8. Gráfica estereográfica de posición y recorrido solar, respecto a la orientación de la fachada principal del inmueble revisado.

Fuente: Elaboración propia con base en software Meteonorm.

La orientación de la fachada hacia el suroeste permite que sea bañada por los rayos del sol entre la 1:00 pm y las 6:00 pm, cuando el azimut ocupa el rango de los $180^{\circ}$ a los $270^{\circ}$.

Sobre los portales y corredores, bioclimáticamente hablando, estos espacios cumplen múltiples funciones, como las de filtro solar, a través de la disposición de elementos como aleros, el de retardo térmico hacia los muros interiores adyacentes a través de limitar la penetración solar, así como la de permitir la libertad de mantener los vanos interiores abiertos, lo que permite que corra aire y se humidifiquen los espacios internos (Gómez y Elías, 2014:33); por lo que la presencia de estos elementos en las viviendas resultan favorables para la mitigación de los efectos del clima cálido, generando así espacios internos habitables y confortables.

\section{USOS TRADICIONALES}

Los usos del espacio habitado, y posterior configuración, son consecuencia de los modos de vida tradicionales, muchos de los cuales se manifiestan hoy en día, en ciertas permanencias arquitectónicas y de costumbres. En esta comunidad, las viviendas han sido parte vinculante entre el habitar y los modos productivos de sus habitantes, así pues, son observables evidencias de esta relación en la apropiación del espacio.

Esta población, al estar aislada de grandes núcleos urbanos, se ha visto obligada a depender y aprovechar los recursos naturales con los que cuenta 
a su alrededor; esta relación con el contexto se manifiesta en la explotación de recursos naturales para satisfacer sus necesidades básicas, así como el aprovechamiento de estos recursos para la adecuación de los espacios interiores en función de sus actividades cotidianas, expresadas éstas en hornos de adobe para la elaboración del tradicional "pan de rancho", zarzos -estructuras ligeras a base de entramados de carrizos, que se cuelgan de los techos- para el secado y almacenamiento de quesos.

Tradicionalmente, la vida al interior de la vivienda se ha desarrollado en función de la familia, con roles de género fuertemente arraigados y claramente identificados, así pues, mientras el hombre sale a trabajar en el campo desde muy temprano, la esposa se encarga del cuidado y atención de la casa y de la familia.

Actualmente, aún siguen vigentes algunas actitudes en el uso del espacio habitacional, como la predilección del uso de la "pila" en detrimento de la lavadora o tarja para las actividades domésticas, la presencia de corrales, huertos y macetas, como también el almacenaje de productos y enseres del campo, como forrajes, herbicidas o monturas de caballo.

En el caso de la vivienda estudiada, se mantiene en la actualidad espacios vigentes o en uso, y espacios residuales que han venido a quedar en desuso, ya sea por la adopción de nuevas formas de habitar, congruentes con el tiempo actual y nuevos estándares sociales, o por la edad de sus moradores.

Actualmente, el portal exterior, es uno de estos espacios que ha venido a perder su función original, la cual históricamente servía como punto de encuentro social y de esparcimiento por parte de sus ocupantes, pues aquí es en donde a determinadas horas del día -tarde-noche- la familia sacaba sillas - por lo menos los miembros más viejos- para ver el acontecer del barrio y conversar con conocidos, familiares o cualquier caminante que pasara; hoy en día esto ya no es observable, quizás por cuestiones de inseguridad, clima, alteración urbana - mayor tránsito y pérdida de paz-, migración a otros núcleos urbanos de los vecinos y congéneres, o incluso debido a la homogeneización de actividades interiores - debido a la inclusión de la televisión en la rutina diaria-en detrimento de actividades al exterior.

La crujía principal, orientada al sur, alberga un billar -uso público-, habitación de huéspedes, -el cual en palabras de la propietaria, es ocupado cuando sobrinos o nietos van a visitarla- y el zaguán, por donde se accede a la vivienda, y que actualmente, en vez de ser un espacio con una función de recibidor está ocupado con muebles de dormitorio - camas, tocadores , buros - que de alguna forma se infiere han sido enseres acumulados durante la vida de los propietarios y los cuales decidieron configurar este espacio a su medida, necesidades y gustos.

Posterior a este recinto, se ubica el portal interior, el cual está dispuesto de forma paralela al área comprendida por el billar y habitación de huéspedes; aquí se encuentran algunos utensilios de cocina y mobiliario doméstico. Este portal, a su vez se encuentra en torno a un patio irregular, en donde abunda una robusta presencia de vegetación, tanto a nivel de piso como en macetas, que ha venido a conformar un microclima interior 
agradable que filtra el sol y limita que se levante polvo, mejorando el nivel de habitabilidad de la vivienda.

Distribuidos sobre esta área descubierta del solar se encuentran algunos espacios con una función predominantemente utilitaria y de servidumbre; núcleo de baños, área de lavado y tendido, acceso de servicio y un tejaban, que en la actualidad sirve como sitio para el almacenaje de enseres del campo y de piezas de materiales de construcción de remplazo -tejas, madera-. Lo anterior permite ejemplificar la forma de apropiación del espacio habitable a través del reconocimiento de los usos que tienen estos, como manifestación cultural de un grupo social, coincidiendo con Beatriz Núñez al referirse que es "necesario reconstruir su hábitat, las prácticas productivas y manifestaciones culturales de sus pobladores (...)" (2017:5), al indagar sobre la cultura y actividades productivas en relación con la tradición en la arquitectura doméstica.

\section{MATERIALES Y SISTEMAS CONSTRUCTIVOS}

En el caso de la vivienda estudiada, la metodología adoptada para la identificación de su constructividad fue la planteada por Dolores Álvarez Gazca, en su texto "El registro de materiales" (Álvarez, 1990), donde se partió del levantamiento de las partes materiales constitutivas del inmueble a través de un registro a partir de una ficha, donde se inició desde la identificación por partidas de cada elemento (figuras 9 y 10).

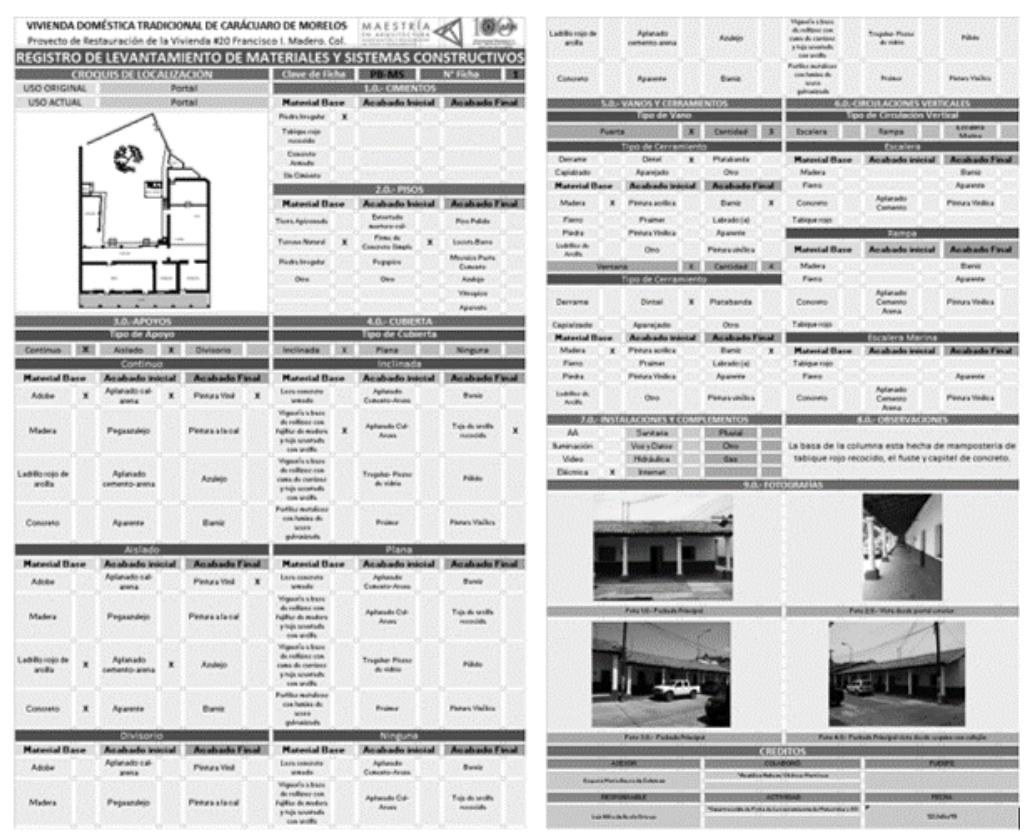

Figuras 9y 10. Ficha de levantamiento de materiales y sistemas constructivos.

Fuente: Elaboración propia en colaboración con Angélica Chávez.

Habiendo realizado lo anterior, se identificaron ciertos patrones constructivos, que, al revisar más viviendas, se corrobora la tradición constructiva de la región. A continuación, se presenta un resumen de los materiales y sistemas constructivos presentes en el inmueble. 
El sistema constructivo consiste en infraestructura conformada de mampostería de piedra irregular - piedra bola, debido a su abundancia en la localidad-, unida con morteros de arcilla y cal, en forma de cimentación corrida. A su vez, la estructura de la cubierta se sostiene sobre apoyos corridos de mampuestos de adobe, con un espesor de $50 \mathrm{~cm}$ y sobre soportes aislados constituidos a base de columnas de concreto con fustes lisos y basamentos de ladrillo rojo recocido. Los muros, con aplanados de cal-arena y pintura a la cal y las columnas están aplanadas con morteros de cemento arena.

La cubierta es inclinada a dos aguas y la estructura está realizada con madera rolliza con entramados de fajillas de madera o cama de carrizos para permitir la penetración de ventilación natural. Se utiliza teja de barro recocido con dimensiones distintas a las existentes actualmente en el mercado.

El principio lógico y de razonamiento detrás de esta arquitectura, desde una mirada de adecuación al medio y de desempeño bioclimático en la construcción es la del resguardo contra la radiación solar y el aprovechamiento de la ventilación, para a través de esta, disipar el calor y aumentar la humedad.

La presencia de portales y corredores que resguardan las zonas habitacionales contiguas, los espesores de muros que evitan la ganancia térmica en verano y retrasan la pérdida en invierno, los intersticios en los muros piñones y los delgados espesores de las cubiertas tradicionales que permiten una renovación y permeabilidad del aire, son algunos ejemplos de respuestas materiales al tema de adecuación al medio, que esta arquitectura regional ha mantenido vigente.

De ahí que esta arquitectura es ligera, abierta, prácticamente sin paredes, donde es más importante la ventilación que la privacidad.

\section{CONCLUSIONES}

Para concluir conviene revisar el porqué de la pérdida paulatina de este patrimonio arquitectónico construido con tierra en el territorio michoacano y de manera especial el de la región de Tierra Caliente.

Cuando se reflexiona sobre la vivienda tradicional, se tiene que considerar la "fragilidad" de ésta ante la influencia de situaciones externas que condicionan la permanencia de un estilo tradicional, que no radica en su estética únicamente, sin que trasciende a elementos intangibles, como modos de vida y tradiciones.

El caso presentado, representa un ejemplo de resistencia cultural, ante los embates de cambio actuales, como son los generacionales, la influencia de los materiales industrializados o la incidencia de poblaciones más desarrolladas, y que si esta vivienda, conserva hoy día parte de sus valores de identidad es en gran parte a que usos y destinos han pervivido a través del tiempo, donde actividades y formas de vida se entremezclan y que la relación con el contexto ha mantenido útil cada elemento constitutivo de la vivienda, manifestando así una correcta adecuación al clima cálido presente. 


\title{
Sólo se puede lograr una ef íżnte proteccón de este patrinonì,
}

\author{
EN LA MEDIDA OUE LAS COMUNIDADES SEAN TOMADAS EN CUENTA \\ $y$ que se promuevan inciativas que micidan en las causas \\ OUE ESTANN ORIGINANDO LOS CAMBIOS FISICOS.
}

Además, sólo se puede lograr una eficiente protección de este patrimonio, en la medida que las comunidades sean tomadas en cuenta y que se promuevan iniciativas que incidan en las causas que están originando los cambios físicos. Se concluye afirmando la necesidad urgente de considerar a los actores sociales como hacedores del patrimonio y reactivar en ellos la memoria, el recuerdo de una habitabilidad del espacio que respondió durante mucho tiempo al contexto físico y social, por supuesto sin negar las necesidades de la vida contemporánea.

\section{FUENTES DE CONSULTA}

Álvarez Gasca, D. (1990), "El registro de materiales”, en La documentación de arquitectura histórica, Universidad de las Américas, México.

Azevedo Salomao ,E. M. (coord.) (2008 a), "La Vivienda Purépecha : historia , habitabilidad, tecnología , y confort de la vivienda purépecha ”, Universidad Michoacana de San Nicolás de Hidalgo, México.

Azevedo Salomao, E. M. (2008b), "Reflexiones en torno a la habitabilidad del espacio". En Blanca Paredes Guerrero (coord.). Memoria IV, Anuario de investigación sobre la conservación, historia y crítica del patrimonio arquitectónico y urbano, Universidad Autónoma de Yucatán, Impresos Itza, pp. 18-21.

Gómez Amador, A., Elías López, P. (2014), “Desempeño térmico de corredores de la casa tradicional de Colima, México", en Revista PALAPA, vol. 2, no. 1 [15], pp. 29-39, [En línea] https :// www .researchgate .net/ publication /272491873_Desempeno _termico_de_corredores_de_la_casa tradicional_de_Colima_Mexico, p.35, consultado el 16 de octubre de 2017.

Gracia, Francisco de (2009), "Morfología de los paisajes". En Entre el paisaje y la arquitectura. Apuntes sobre la razón constructiva, Donostia-San Sebastián, Editorial Nerea.

Guzmán Barriga, C. (coord.) (2007), Michoacán guía de arquitectura y paisaje, Ed. Bilingüe, Gobierno Constitucional del Estado de Michoacán , Sevilla, Consejería de Obras Públicas y Transportes, Dirección General de Arquitectura y Vivienda, México.

Herrera Peña, J. (1995), Maestro y discípulo, Universidad Michoacana de San Nicolás de Hidalgo, Secretaría de Difusión Cultural, México.

Instituto Nacional de Estadística y Geografía (INEGI) (2009), Prontuario de información geográfica municipal de los Estados Unidos Mexicanos. Carácuaro, Michoacán de Ocampo, 16013, [En línea] https://caracuaro.com/documentos/pdf/prontuario-deinformacion-geografica.pdf, consultado el 23 de mayo de 2019.

Núñez Miranda, B. (2013), “Tradiciones, formas y vivienda en Tonalá”, en García Fernández, E., Vaca, A. y Azevedo Salomao, E. M. (coords.), 
Espacios habitables, memoria y construcción del patrimonio, El Colegio de Jalisco, Zapopan, pp. 187-209.

Ochoa Cabrera, A. (2003), "Tipología de la arquitectura habitacional tradicional en la región sureste de la tierra caliente de Michoacán 1940-2003", en Memorias del 1er Seminario de Arquitectura, Territorio, y Población en el antiguo Obispado de Michoacán, Época Virreinal, Universidad Michoacana de San Nicolás de Hidalgo, Consejo Nacional de Ciencia y Tecnología, Morelia.

Sun Earth Tools, [En línea] https://www.sunearthtools.com/es/index.php, consultado el 07 de octubre de 2019.

Torres Garibay, L. A. (2008), "Coincidencias, permanencias y técnica en la constructividad. Observaciones a través de la arquitectura vernácula”. En Blanca Paredes Guerrero (coord.). Memoria IV, Anuario de investigación sobre la conservación, historia y crítica del patrimonio arquitectónico y urbano, Universidad Autónoma de Yucatán, Impresos Itza, Mérida, pp. 26-28. 